\title{
Synthesis of damnacanthal, a naturally occurring 9,10-anthraquinone and its analogues, and its biological evaluation against five cancer cell lines.
}

\begin{abstract}
Damnacanthal and nordamnacanthal, two naturally occurring 9,10-anthraquinones, and their analogues were synthesized. Cytotoxic activity against five cancer cell lines was evaluated using MTT assay. 2-Bromomethyl-1,3-dimethoxyanthraquinone was found to display the highest activity against all cell lines with IC50 range of $2-8 \mu \mathrm{M}$. Structure-activity relationship (SAR) assessment was considered to rationalise the cytotoxic effect. Bromomethyl group at position C-2 of the anthraquinone was found to be important in exerting cytotoxic activity of this class of compounds. The presence of the flanking methoxyl or hydroxyl groups at C-1 and C-3 also contributes to this activity. Finally, the antioxidant effect of these compounds was evaluated. MTT assay was used to measure the cytotoxicity against different cancer cell lines. Antioxidant activity was measured by FTC and TBA methods. Only two anthraquinones, damnacanthal and nordamnacanthal, were found to be antioxidative.
\end{abstract}

Keyword: Anthraquinones; Synthesis; Cytotoxicity; Antioxidant activity. 